\title{
INSIDE DISCRETE-EVENT SIMULATION SOFTWARE: HOW IT WORKS AND WHY IT MATTERS
}

\author{
Thomas J. Schriber \\ The University of Michigan \\ Business Information Technology \\ Ann Arbor, MI 48109-1234, USA
}

\author{
Daniel T. Brunner \\ Kiva Systems, Inc. \\ 225 Wildwood Avenue \\ Woburn, MA 01801-2025, U.S.A.
}

\begin{abstract}
This paper provides simulation practitioners and consumers with a grounding in how discrete-event simulation software works. Topics include discrete-event systems; entities, resources, control elements and operations; simulation runs; entity states; entity lists; and entity-list management. The implementation of these generic ideas in AutoMod, SLX, and ExtendSim is described. The paper concludes with several examples of "why it matters" for modelers to know how their simulation software works, including discussion of examples covering AutoMod, SLX, and ExtendSim, and also SIMAN (Arena), ProModel, and GPSS/H.
\end{abstract}

\section{INTRODUCTION}

In this section we discuss the motivation for developing this paper, and comment on the paper's structure and the terminology and conventions used in the paper.

\subsection{Background}

A "black box" approach is often taken in teaching and learning discrete-event simulation software. The external characteristics of the software are studied, but the foundation on which the software is based is ignored or is touched on only briefly. (This might be attributable to a lack of time or of appropriate written material.) Choices made in implementation of the foundation might not be studied at all and related to step-by-step model execution. The modeler therefore might not be able to think things through when faced with such needs as developing good approaches for modeling complex situations, using interactive tools to come to an understanding of error conditions arising during model development, and using interactive tools to verify that complex system logic has been modeled correctly. The objective of this paper, then, is to describe the logical underpinnings of discrete-event simulation and illustrate this material in terms of various implementations of discrete-event simulation software.

This paper is a revised version of an identically named paper from the 1996 Winter Simulation Conference (Schriber and Brunner 1996). The 1996 paper covered the entity-list management rules and "why it matters" for SIMAN (the language underlying Arena), ProModel, and GPSS/H. A substantially expanded version of the 1996 paper containing figures, flow charts, and additional explanation can be found in Schriber and Brunner (1998).

\subsection{Structure of the Paper}

In Sections 2, 3 and 4 we comment on the nature of discrete-event simulation; basic simulation constructs such as entities, resources, control elements, and operations; and model execution. Sections 5 and 6 deal with entity states and entity management data structures. Section 7 discusses three specific implementations of entity management rules. Section 8 explores various aspects of "why it matters." 


\section{Schriber and Brunner}

\subsection{Terminology and Conventions}

Throughout this paper we use terms that we define as well as terms reserved by the developers of particular simulation tools. Terms we define are boldfaced on first use. Tool-specific terms are Capitalized or, where appropriate, are spelled out in ALL CAPS.

\section{ABOUT DISCRETE-EVENT SIMULATION}

This section introduces the transaction-flow world view that serves as the basis for the paper, and then discusses the nature of discrete-event simulation and the logical challenges inherent in developing discrete-event simulation languages.

\subsection{The Transaction-Flow World View}

The "transaction-flow world view" often provides the basis for discrete-event simulation. In this world view, a system is visualized as consisting of discrete units of traffic that move ("flow") from point to point in the system while competing with each other for the use of scarce resources. The units of traffic are sometimes called "transactions," giving rise to the phrase "transaction flow."

Numerous systems fit the preceding description. Included are manufacturing, material handling, transportation, health care, civil, natural resource, communication, defense, and information processing systems, and queuing systems in general.

\subsection{The Nature of Discrete-Event Simulation}

A discrete-event simulation is one in which the state of a model changes at only a discrete, but possibly random, set of simulated time points, called event times. Two or more traffic units often have to be manipulated at one and the same time point. Such "simultaneous" movement of traffic at a time point is achieved by manipulating units of traffic serially at that time point. This often leads to logical complexities in discrete-event simulation because it raises questions about the order in which two or more units of traffic are to be manipulated at a given simulated time.

\subsection{Discrete-Event Modeling Languages}

The challenges faced by a modeler escalate for the designer of a modeling language. The designer must take the logical requirements of discrete-event simulation into account in a generalized way. Choices and tradeoffs exist. As a result, although discrete-event simulation languages are similar in broad terms, they can differ in subtle but important particulars.

\section{ENTITIES, RESOURCES, CONTROL ELEMENTS, AND OPERATIONS}

The term entity is used here to designate a unit of traffic (a "transaction"). Entities instigate and respond to events. An event is a happening that changes the state of a model (or system). In a model of an order-filling system, for example, the arrival of an order, which is an event, might be simulated by bringing an entity into the model.

There are two possible types of entities, here referred to as external entities and internal entities. External entities are those whose creation and movement is explicitly arranged for by the modeler. In contrast, internal entities are created and manipulated implicitly by the simulation software itself. For example, internal entities might be used in some languages to simulate machine failures, whereas external entities might be used to simulate the use of machines.

The term resource designates a system element that provides service (such as a drill, an automated guided vehicle, or space in an input buffer). The users of resources are usually entities. (For example, a work-in-process entity claims space in an input buffer, then captures an automated guided vehicle to move it to the input buffer.) Resources are usually capacitylimited, so entities compete for their use and sometimes must wait to use them, experiencing delay as a result.

The term control element designates a construct that supports other types of delay or logical alternatives based on a system's state. Control elements can take the form of switches, counters, user data values, and system data values built into the modeling tool. Complex control may rely on truth-valued expressions that use arithmetic and/or Boolean combinations of control elements.

An operation is a step carried out by or on an entity while it moves through a system. The operations applicable to a ship at a harbor might be these: arrive at the harbor; request a berth; capture a berth; request a tugboat; capture a tugboat; get pulled into the berth; free the tugboat; load cargo; request a tugboat; get pulled out of the berth; free the berth; get pulled into open water; free the tugboat; depart. 


\section{Schriber and Brunner}

\section{OVERVIEW OF MODEL EXECUTION}

We now review the concepts of experiments, then replications, and then simulation runs, concluding this section with discussion of the anatomy of a run.

\subsection{Experiments, Replications, and Runs}

A simulation project is comprised of experiments. Experiments are differentiated by the use of alternatives in a model's logic and/or data. An alternate part-sequencing rule might be tried, for example, in the model of a production system, and/or the quantity of various types of machines might be varied. Or the number of loading and unloading berths in a harbor might be varied.

Each experiment consists of one or more replications (trials). A replication is a simulation that uses the experiment's model logic and data but its own unique set of random numbers, and so produces unique statistical results that can be analyzed in a set of such replications.

A replication consists of initializing the model, running it until a run-ending condition is met, and reporting results. This "running it" phase is called a run.

\subsection{Inside a Run}

During a run the simulation clock (an internally managed, stored data value) tracks the passage of simulated time (as distinct from wall-clock time). The clock advances (automatically) in discrete steps (typically of unequal size) during the run. After all possible actions have been taken at a given simulated time, the clock is advanced to the time of the next earliest event. Then the appropriate actions are carried out at this new simulated time, etc.

In essence, the execution of a run therefore takes the form of a two-phase loop: "carry out all possible actions at the current simulated time," followed by "advance the simulated clock," with these two phases repeated again and again until a runending condition (usually modeler-specified) comes about. The two phases are respectively called the Entity Movement Phase (EMP) and the Clock Update Phase (CUP) here.

\section{ENTITY STATES}

Entities migrate from state to state while they work their way through a model. There are five entity states, as detailed below.

\subsection{The Active State}

The Active State is the state of the currently moving entity. Only one entity moves at any instant of wall-clock time. This entity moves until it encounters a delay of one type or other. It then migrates to an alternative state. Some other entity might then become the next active entity at that simulated time. (See the Ready State.) And so on.

\subsection{The Ready State}

During an Entity Movement Phase there may be more than one entity ready to move, and yet entities can only move (be in the Active State) one-by-one. The Ready State is the state of entities waiting to enter the Active State during the current Entity Movement Phase.

\subsection{The Time-Delayed State}

The Time-Delayed State is the state of entities waiting for a known future simulated time to be reached so that they can then (re)enter the Ready State. A "part" entity is in a Time-Delayed State, for example, while waiting for the future simulated time at which an operation being performed on it by a machine will come to an end.

\subsection{The Condition-Delayed State}

The Condition-Delayed State is the state of entities delayed until some specified condition comes about, e.g., a "part" entity might wait in the Condition-Delayed State until its turn comes to use a machine. Condition-Delayed entities are transferred automatically from the Condition-Delayed state to the Ready State when conditions permit. 


\section{Schriber and Brunner}

\subsection{The Dormant State}

Sometimes it is desirable to put entities into a state from which no escape will be triggered automatically by changes in model conditions. We call this state the Dormant State. Dormant-State entities rely on modeler-supplied logic to transfer them from the Dormant State to the Ready State. Job-ticket entities might be put into a Dormant State, for example, until an operator entity decides which job-ticket to pull next, with consequent transfer of the job ticket to the Ready State.

\section{ENTITY MANAGEMENT STRUCTURES}

In our generic model, simulation software uses the following lists to organize entities in the five entity states.

\subsection{The Active Entity}

The active entity is resident on an unnamed "list" consisting only of the active entity. The Active-State entity moves nonstop until encountering an (attempted) step that causes it to migrate into another entity state (transfers it to another list) or removes it from the model. A Ready-State entity then becomes the next Active-State entity. Eventually there are no more Ready-State entities at the current time. The EMP then ends and a Clock Update Phase begins.

\subsection{The Current Events List}

Entities in the Ready State are kept in a single list we call the current events list (CEL). Entities migrate to the CEL from the future events list, from delay lists, and from user-managed lists. (Each of these latter lists is described below). In addition, entities (if any) cloned from the Active-State entity usually start their existence on the CEL.

CEL Entities are generally ranked in FIFO order. Some software tools provide a built-in entity Priority attribute used to group Entities on the CEL in priority order.

\subsection{The Future Events List}

Entities in the Time-Delayed State belong to a single list into which they are inserted at the beginning of their time-based delay. This list, called the future events list (FEL) here, is usually ranked by increasing entity move time. (Move time is the simulated time at which an entity is scheduled to try to move again.) At the time of entity insertion into the FEL, the entity's move time is calculated by adding the value of the simulation clock to the known (sampled) duration of the time-based delay.

After an Entity Movement Phase is over, the Clock Update Phase sets (advances) the clock's value to the move time of the FEL's highest ranked (smallest move time) entity. This entity is then transferred from the FEL to the CEL, migrating from the Time-Delayed State to the Ready State and setting the stage for the next EMP to begin.

The preceding statement assumes there are not other entities on the FEL whose move time matches the clock's updated value. In the case of move-time ties, some tools will transfer all the time-tied entities from the FEL to the CEL during a single CUP, whereas other tools take a "only one entity transfer per CUP" approach.

Languages that provide internal entities usually use the FEL to support the timing requirements of these entities. The FEL is then typically comprised both of external and internal entities in such languages.

\subsection{Delay Lists}

Delay lists (there can be many) are lists of entities in the Condition-Delayed State. These entities are waiting (e.g., waiting their turn to use a machine) for the time when their delay is resolved so they can be transferred automatically into the Ready State on the CEL. Delay lists, which are generally created automatically by the simulation software, are managed by using related waiting or polled waiting.

If a delay can be related easily to model events that might resolve the delay, then related waiting can be used to manage the delay list. For example, suppose a machine's status changes from busy to idle. In response, the software can automatically remove the next waiting entity from the appropriate delay list and put it in the Ready State on the current events list. Related waiting is the prevalent approach used to manage conditional delays.

If the delay condition is too complex to be related easily to events that might resolve it, polled waiting can be used. With polled waiting the software checks routinely to see if entities can be transferred from one or more delay lists to the Ready State. Complex delay conditions for which polled waiting can be useful include Boolean combinations of state changes, e.g., a berth is empty and a tugboat is idle. 


\subsection{User-Managed Lists}

User-managed lists (there can be many) are lists of entities in the Dormant State. The modeler must take steps to establish such lists and usually must provide the logic needed to transfer entities to and from the lists. (Except for very simple one-line, one-server service points in a system, the underlying software has no way to know why entities have been put into usermanaged lists in the first place, and therefore has no plausible basis for automatically removing entities from such lists.)

\section{IMPLEMENTATION IN THREE TOOLS}

The tools chosen here for commentary on implementation particulars are AutoMod (Phillips 1997); SLX (Henriksen 2000; Schulze 2008); and ExtendSim (Diamond et al. 2007; Krahl and Lamperti 1997). A previous version of this paper (Schriber and Brunner 1996) covered SIMAN (Kelton 2009; Pegden, Shannon and Sadowski 1995), ProModel (ProModel Corporation 1995), and GPSS/H (Henriksen and Crain 2000) in similar detail. (Section 8 presents examples of "why it matters" for each of the six tools.) These six are among more than forty tools reported in 2007 for discrete-event simulation (Swain 2007). We think these tools are representative, but they clearly are not exhaustive.

\subsection{AutoMod}

AutoMod equivalents for the preceding generic terms are given in Table 1. For example, AutoMod uses Actions to specify operations for Loads.

Table 1: AutoMod Terminology

\begin{tabular}{|l|l|}
\hline Generic Term & AutoMod Equivalent \\
\hline External Entity & Load \\
\hline Internal Entity & Logical Load \\
\hline Resource & Resource; Queue; Block \\
\hline Control Element & $\begin{array}{l}\text { Counter; } \\
\text { Process Traffic Limit }\end{array}$ \\
\hline Operation & Action \\
\hline Current Events List & Current Event List \\
\hline Future Events List & Future Event List \\
\hline Delay List & $\begin{array}{l}\text { Delay List; } \\
\text { Condition Delay List; } \\
\text { Load Ready List }\end{array}$ \\
\hline User-Managed List & Order List \\
\hline
\end{tabular}

\subsubsection{The Current Event List}

The current events list is named the Current Event List in AutoMod. Cloned Loads, Loads leaving the Future Event List due to a clock update, and Loads ordered off Order Lists are placed immediately on the CEL. The insertion rule is to rank first by priority (priority is a built-in attribute of every Load) and then FIFO within priority.

When the CEL becomes empty, the Condition Delay List (see below) is checked, and Loads may be transferred from there to the CEL. This continues until the CEL is empty and no more Loads can be transferred, at which point the EMP is over and a CUP is initiated.

\subsubsection{The Future Event List}

The AutoMod Future Event List (FEL) is like future events lists in other tools. Loads arrive on the FEL in the Time-Delayed State by executing a WAIT FOR statement. AutoMod allows the specification of time units (day, hr, min, sec) in a WAIT FOR statement.

The AutoMod CUP removes multiple Loads from the FEL if they are tied for the earliest move time, inserting them one by one into their appropriate place on the CEL.

There are also internal entities in AutoMod, called Logical Loads, that do things such as wait on the FEL to trigger scheduled shift breaks. 


\section{Schriber and Brunner}

\subsubsection{Delay Lists}

Delay Lists (DL's) are lists of Loads waiting to claim capacity provided by a finite-capacity element (a resource, i.e., Resource, Queue, Block; or a control element, i.e., Counter, or Traffic Limit Process). Each finite capacity element within the model has a DL associated with it.

The waiting that results in these five cases is related waiting. Whenever capacity is freed, one Load from the head of the element's DL is tentatively placed on the CEL (but a placeholder is left on the DL). When that Load is encountered during the EMP, it tries to claim the requested capacity. If it fails (for example because it wants two units but only one is free), it is returned to the DL in its original place.

Immediately after this evaluation, if there is still any unused capacity, the next Load (if any) on the DL is placed on the CEL. Processing of the active Load then continues. After each time a tentatively placed Load is evaluated during the EMP, the existence of available capacity will cause another Load to be removed from the DL.

\subsubsection{The Condition Delay List}

For conditional waiting apart from the five cases described above, AutoMod has a WAIT UNTIL statement that results in polled waiting. WAIT UNTIL conditions can be compounded using Boolean operators. If a Load executes a WAIT UNTIL and the condition is false, the Load is placed on a single global AutoMod list called the Condition Delay List (CDL).

After the Current Events List has been emptied, but before the simulation clock is updated, all Loads on the Condition Delay List are moved to the Current Events List (actually, the Condition Delay List "becomes" the Current Events List) if there has been a state change for at least one element of the same general type (e.g. Queue) for which any Load on the Condition Delay list is waiting. (This mechanism is primarily "polled," where the polling process is triggered by a change in state of at least one element of the same general type.)

If the Current Events List is now non-empty, the Entity Movement Phase resumes. If the condition for which a CEL Load is waiting is not yet satisfied, AutoMod moves that Load from the Current Events List back to the Condition Delay List. The Condition Delay List in some cases may be emptied multiple times during one Entity Movement Phase until eventually the Current Events List has been emptied without having triggered a state change related to any Load on the Condition Delay List. A Clock Update Phase then occurs.

Because of the potential for repetitive list migration with WAIT UNTIL, AutoMod's vendor encourages the use of Order Lists or other explicit control mechanisms to manage complex waiting.

\subsubsection{Order Lists}

AutoMod implements the Dormant State with Order Lists, which are user-managed lists of Loads. After a Load puts itself onto an Order List (by executing a WAIT TO BE ORDERED Action), it can only be removed by another Load (or another active model element such as a Vehicle) which executes an ORDER Action. An ORDER Action may specify a quantity of Loads, or a condition that must be satisfied for a given Load if that Load is to be ordered, or both. Loads successfully ordered are placed immediately on the CEL (one at a time according to how they were chosen from the Order List, and ranked on the CEL by priority (with priority ties resolved FIFO).

Order Lists can achieve performance improvements over CDL waiting because Order Lists are never scanned except on explicit request.

AutoMod Order Lists offer several interesting wrinkles, including: the ability for an ordering Load to place a back order if the ORDER quantity is not satisfied; the ability for a Load on an Order List to be ordered to continue to the next Action instead of to a Process (this feature is useful for control handshaking); and the ability to have a function called for each Load on the Order List (by using the ORDER...SATISFYING Action).

\subsubsection{Other Lists}

AutoMod has a number of material handling constructs that are integrated with Load movement. For vehicle systems there are three other types of lists. Loads on Load Ready Lists (LRL) (one list per vehicle system) are waiting to be picked up by a vehicle. Loads claimed (but not yet picked up) by a vehicle reside on the vehicle's Vehicle Claim List (VCL). Claimed loads that have been picked up reside on the vehicle's Vehicle Onboard List (VOL). The vehicle then becomes the active "load" and moves among AutoMod's lists (FEL, CEL, and possibly DL's) rather than the Loads themselves. 


\subsection{SLX}

SLX is a hierarchical language in which the built-in primitives are at a lower level than most simulation languages, facilitating user (or developer) definition of the behavior of many system elements. This design philosophy allows the SLX developer to create higher-level modeling tools whose constructs have precisely defined modifiable behavior.

Equivalents for the generic terms for users of low-level SLX are given in Table 2. For example, SLX uses Control Variables to act as Control Elements. The "control" modifier can be attached to a Variable of any data type (integer, real, string, etc.). A Control Variable can be global, or it can be a local Variable declared in an Object's Class definition. (A Classdeclared Variable is analogous to an attribute in other tools.)

Note that SLX has two types of Objects: Active and Passive. An Active Object is distinguished from a Passive Object by the presence of actions - executable Statements - in an Active Object's Class definition. (Even without actions, Passive Objects are useful in their own right, functioning as user-defined complex data structures.)

Table 2: SLX Terminology (Low-level)

\begin{tabular}{|l|l|}
\hline Generic Term & SLX Equivalent \\
\hline External Entity & Active Object and its Puck(s) \\
\hline Internal Entity & none \\
\hline Resource & Control Variable \\
\hline Control Element & Control Variable \\
\hline Operation & Statement \\
\hline Current Events List & Current Events Chain \\
\hline Future Events List & Future Events List \\
\hline Delay List & Delay List \\
\hline User-Managed List & Set (see section 7.2.4) \\
\hline
\end{tabular}

Table 3 shows how higher-level tools based on SLX might exploit the definitional capabilities of SLX.

Table 3: Tools Based on SLX

\begin{tabular}{|l|l|}
\hline Generic Term & SLX Equivalent \\
\hline Resource & Active or Passive Object \\
\hline Control Element & Active or Passive Object \\
\hline Operation & User-defined Statement \\
\hline Delay List & User-defined (based on Set) \\
\hline User-Managed List & User-defined (based on Set) \\
\hline
\end{tabular}

\subsubsection{The Current Events Chain}

The current events list is named the Current Events Chain (CEC) in SLX. The members of the CEC are given the interesting name Puck.

What is a Puck? SLX dissociates the concept of an Active Object (with its associated local data) from a Puck, which is the "moving entity" that executes the actions, carries its own entity scheduling data, and migrates from list to list. The effect of this dissociation is that a single Object can "own" more than one Puck. All Pucks owned by a single Object share the Object's local data (attributes). For example, one application of this "local parallelism" feature (as compared with the "global parallelism" offered by "clone" or "split" actions in other languages) is the use of a second Puck to simulate a balk time while the original Puck is waiting for some condition. (If the condition comes about before the balk time has elapsed, no balking occurs; otherwise, balking does occur.)

Activating a new Object creates one Puck and launches that Puck into action. In many cases no additional Pucks are ever created, and the combination of an Active Object and its Puck forms the equivalent of an entity in the terminology of this paper. (Passive Objects have no actions and therefore own no Pucks.)

Newly activated Pucks, Pucks leaving the FEL due to a clock update, and reactivated Pucks (see 7.2.4) are placed immediately on the CEC. The CEC is ranked FIFO by priority. The SLX CEC is empty when an EMP ends. 


\section{Schriber and Brunner}

\subsubsection{The Future Events List}

The SLX Future Events List (FEL) is like future events lists in other tools. Pucks arrive on the FEL in the Time-Delayed State by executing an ADVANCE statement.

The SLX CUP will remove multiple Pucks from the FEC if they are tied for the earliest move time, inserting them one by one into their appropriate place on the CEC.

Because the SLX kernel functionality does not include downtimes or even repetitive Puck generation (scheduled arrivals), all activity on the SLX FEL unfolds as specified by the developer of the SLX model. More generally, if a user is using a model (or is using a model builder) that contains higher-level primitives defined by a developer, chances are that all kinds of things are going on behind the scenes, hidden from the higher-level user's view.

\subsubsection{Delay Lists}

Delay Lists (DL's) are lists of Pucks waiting (via WAIT UNTIL) for state changes in any combination of Control Variables and the simulation clock value. A Puck waiting for a compound condition involving two or more Control Variables is listed on more than one DL. All higher-level constructs defined by developers can use this mechanism. Each Control Variable (which may be a local Variable, in which case there is one for each Object in the Class) has a separate DL associated with it.

A DL is ranked by order of insertion. All pucks on a DL are removed whenever the associated Control Variable changes value and are inserted one at a time into the CEC. Removed Pucks that are waiting on compound conditions are also tentatively removed from each of the other Delay Lists to which they belong. As these Pucks are encountered on the CEC during the EMP, those failing to pass their WAIT UNTIL are returned to the Delay List(s) for those Control Variables still contributing to the falseness of the condition.

For conditions that include a clock reference, the Puck is inserted if necessary into the FEL, subject to early removal from the FEL if the condition becomes true due to other Control Variable changes.

This low-level related waiting mechanism based on Control Variables is the default SLX approach to modeling all types of simple or compound Condition-Delayed states.

\subsubsection{Sets and User-Managed Waiting}

SLX handles the Dormant State in a unique way. Instead of moving the Puck from the active state to a user-managed list and suspending it, all in the same operation, SLX breaks this operation into two pieces.

First, the Puck usually joins a Set. But joining a Set does not automatically suspend the Puck. A Puck can belong to any number of Sets. Set membership merely provides any other Puck with access to the member Pucks.

To go into the Dormant state, a Puck executes a WAIT statement. It then is suspended indefinitely, outside of any particular list, until another Puck identifies the waiting Puck and executes a REACTIVATE statement for it. Often this other Puck is scanning a Set to find the Puck to REACTIVATE, but a Set is not exactly the same as a user-managed list in our terminology. A Dormant-state Puck might be a member of no Sets (as long as a pointer to it has been stored somewhere) or of one or more Sets.

An SLX developer can easily define a user-managed list construct, using Sets, WAIT, and REACTIVATE as building blocks, that mimics those of other languages or offer unique features of its own.

\subsection{ExtendSim}

ExtendSim (originally named Extend) uses a message-based architecture for discrete-event simulation. Various types of messages are used to schedule events, propel Items (Entities) through a model, enforce the logic incorporated into a model, and force computation. The senders and receivers of messages are Blocks (Operations), including the Executive Block (master controller). In ExtendSim, it is Block execution that is scheduled. (When a Block executes, for example, this can trigger the sending of messages back and forth among Blocks, with the effect of logically propelling an Item along its Block-based path in a model.)

ExtendSim equivalents for the terms introduced in the earlier generic discussion are summarized in Table 4.

\subsubsection{Blocks}

Blocks are ExtendSim's basic modeling construct. Each Block has an icon, message-passing connectors, dialog capability, and behavior-defining code. Residence Blocks can hold Items while simulated time goes by, whereas Passing Blocks cannot. 


\section{Schriber and Brunner}

(Items go through Passing Blocks in zero simulated time.) Models can be constructed by selecting pre-programmed Blocks from ExtendSim's Block libraries. The modeler can also modify the source code given for library Blocks. (All Blocks in the base version of ExtendSim are open source.) Finally, the modeler can create customized Blocks from scratch (userprogrammed Blocks) using development tools that ExtendSim provides.

Table 4: ExtendSim Terminology

\begin{tabular}{|l|l|}
\hline Generic Term & ExtendSim Equivalent \\
\hline External Entity & Item \\
\hline Internal Entity & none \\
\hline Resource & $\begin{array}{l}\text { Resource; Resource Pool; } \\
\text { generally, any Block with a } \\
\text { limited capacity }\end{array}$ \\
\hline Control Element & Block Dialog \\
\hline Operation & Block \\
\hline Current Events List & $\begin{array}{l}\text { Next Times Array } \\
\text { and Current Events Array }\end{array}$ \\
\hline Future Events List & Time Array \\
\hline Delay List & $\begin{array}{l}\text { List of Items Resident in a } \\
\text { Pre-Programmed Block }\end{array}$ \\
\hline User-Managed List & $\begin{array}{l}\text { List of Items Resident in a } \\
\text { User-Programmed Block }\end{array}$ \\
\hline
\end{tabular}

\subsubsection{The Time Array}

ExtendSim uses a Time Array to schedule future Block executions. For a given model, the Time Array contains one or more elements for each Block. A Time Array element records the future time for which execution of that Block has been scheduled. (The potential for a Block to have more than one Time Array element is an enhancement in Version 7 of the language. This feature can be useful when a Block has multiple, dissimilar events, as for example in conveyor modeling.)

Blocks not currently scheduled for future execution are temporarily "blacked out" by recording arbitrarily large time values for them in the Time Array.

Residence Blocks that can hold multiple Items manage the corresponding event times internally, with only the earliest of the Block's event times kept in the Time Array.

Block execution can result in scheduling future Block executions. For example, if messages are passed that result in an Item entering a unit-capacity Residence Block designed to hold the Item until a sampled amount of simulated time has elapsed, then the Time Array entry for that Block will have its value set accordingly.

The number of Blocks in a given model is constant, which means the Time Array is of fixed and relatively small size. Because of its small size, the Time Array is searched to find imminent event time; it is not kept in sort order. This makes it straightforward for a Block to change its event time because no searching of the event list is required.

\subsubsection{The Next Times and Current Events Arrays}

The Next Times Array is used to manage the execution of Blocks whose execution has been scheduled via the Time Array. The Next Times Array is populated just prior to a Block Execution Phase (ExtendSim's equivalent of an Entity Movement Phase) as follows. At each Clock Update Phase, the Time Array is searched to find the earliest future time at which a Block execution has been scheduled. Identifiers for the corresponding Block (or Blocks, in case of time ties) is (or are) then put into the Next Times Array. The Block Execution Phase (BEP) then begins, with the Executive messaging the most highly qualified Block in the Next Times array to start its execution.

The Current Events Array is used to manage the resumption of execution of Blocks whose execution has been temporarily suspended during the course of a Block Execution Phase. For example, suppose a Block sends a message, and the receiving Block replies (returns control) immediately to the sending Block (even though the receiving Block still has to do additional processing at the simulated time in question). In this case, the receiving Block's identifier is added to the Current Events Array. When the sending Block is finished executing, the Executive sends a message to the most highly qualified Block in the Current Events Array to resume its execution. Eventually, the Current Events Array becomes empty. Then the Executive turns again to the Next Times Array, sending a message to the most highly qualified Block to start executing. 


\section{Schriber and Brunner}

During a Block Execution Phase, it is possible for Blocks to schedule themselves to be executed at the current simulated time (that is, during the ongoing BEP). The Current Events Array comes into play here, too, to manage the execution of Blocks in such cases.

For example, if a capacity-constrained Block becomes non-full as a result of some other Block's execution, the non-full Block puts its identifier into the Current Events Array. The Executive will later (but at the same simulated time) send a message to the Block to start executing. The Block will then try to pull into itself Items (if any) that have been waiting to enter the Block. (In ExtendSim, Items can be both pulled and pushed through a model.)

When the Current Events Array and the Next Times Array both become empty, this brings ExtendSim's Block Execution Phase to an end. Then the next CUP and BEP take place, repeating until a simulation-ending condition is satisfied.

\subsubsection{Delay Lists}

Delay lists are comprised of Items delayed in Residence Blocks, waiting their turn to be pulled or pushed into their next Block(s). Message passing is used to accomplish the pulling and pushing when model conditions permit. ExtendSim provides related-waiting management of delay lists based on user-specified FIFO, LIFO, Priority, Attribute, Reneging, and Matching alternatives.

Waiting for the resolution of compound conditions is normally achieved in ExtendSim by appropriately combining Blocks and exploiting ExtendSim's message-based architecture. We view this here as a form of related waiting, because it is a change in an underlying value that triggers a re-evaluation of the condition that brought about the waiting in the first place.

Because of ExtendSim's messaging architecture, polled waiting is generally not necessary. A message is sent when a value changes and any conditions are evaluated at that moment. Waiting for a clock based event can be achieved by using a Block that schedules events, e.g., Shift; Lookup Table; Equation. These Blocks send a message at scheduled times. Polled waiting is available, however, with use of the Gate block and selection of its "Check demand at each event" option.

\subsubsection{User-Managed Lists}

The modeler can work with user-programmed Blocks to create and manage lists of the modeler's own design. The code for custom blocks can be written to achieve the modeler's objectives in this regard, just as the code for ExtendSim's preprogrammed Blocks has been written to specify the behavior of those Blocks. ExtendSim provides functions that can be used by Blocks to share lists (arrays) with other Blocks, further supporting customized list management in models.

\section{WHY IT MATTERS}

In sections 8.1-8.5 we describe situations that reveal some practical differences in implementation particulars among SIMAN, ProModel (Version 3), GPSS/H, AutoMod, SLX, and ExtendSim. These differences reflect differing implementation choices made by the software designers.

None of the alternative approaches mentioned is either intrinsically "right" or "wrong." The modeler simply must be aware of the alternative in effect in the simulation software being used and work with it to produce the desired outcome. (If a modeler is unaware of the alternative in effect, it is possible to mis-model a situation and perhaps not become aware of this mis-modeling.)

In section 8.6, we comment on how knowledge of software internals is needed to make effective use of software checkout tools.

Finally, in section 8.7, we point out that knowledge of internals aids in understanding performance monitoring.

\subsection{Trying to Re-capture a Resource Immediately}

Suppose a job in a flexible job shop releases a machine (for which other less qualified jobs are waiting), then decides as its next step to re-capture that machine. Will the job be able to re-capture the machine immediately?

Of interest here is the order of events following the giving up of a resource. There are at least three alternatives: (1) Coupled with the giving up of the resource is the immediate choosing of the next user of the resource, without the releasing entity having yet become a contender for the resource. (2) The choosing of the next user of the resource is deferred until the releasing entity has become a contender. (3) "Neither of the above;" that is, without paying heed to other contenders, the releasing entity recaptures the resource immediately. 


\section{Schriber and Brunner}

SIMAN and ExtendSim implement (1). ProModel implements (2). GPSS/H and AutoMod implement (3) by default. In SLX, using a low-level Control Variable as the resource state, the result is also (3). (However, developers could implement higher-level resource constructs in SLX that behave in any of the three ways.)

\subsection{The First in Line is Still Delayed}

Suppose two Condition-Delayed entities are waiting in a delay list because no units of a particular resource are idle. Suppose the first entity needs two units of the resource, whereas the second entity only needs one unit. Now assume that one unit of the resource becomes idle. The needs of the first list entity cannot yet be satisfied, but the needs of the second entity can. What will happen?

There are at least three possible alternatives: (1) Neither entity claims the idle resource unit. (2) The first entity claims the one idle resource unit and waits for a second unit. (3) The second entity claims the idle resource unit and migrates to the Ready State.

As in Section 8.1, each of these alternatives comes into play in the tools considered here. SIMAN (SEIZE) and ProModel (GET or USE) implement (1) and (2) respectively, by default. AutoMod (GET or USE), GPSS/H (ENTER or TEST), and SLX (WAIT UNTIL on a Control Variable) implement (3) by default. ExtendSim also implements (3) by default. But ExtendSim gives the modeler the choice of locally implementing (1) for resources specified by the modeler. The modeler does this by checking an "Only allocate resource pool to the highest ranked Item" option for each such resource.

\subsection{Yielding Control Temporarily}

Suppose the active entity wants to give control to one or more Ready-State entities, but then needs to become the active entity again before the simulation clock has been advanced. This scenario might come into play, for example, if the active entity has opened a switch permitting a set of other entities to move past a point in the model, and then needs to re-close the switch after the forward movement of the other entities has been accomplished. (Perhaps a group of identically flavored cartons of ice cream is to be transferred from an accumulation point to a conveyor leading to a one-flavor-per-box packing operation.)

In SIMAN and AutoMod, the effect can be accomplished approximately with a DELAY (SIMAN) or WAIT FOR (AutoMod) that puts the active entity into a Time-Delayed State for an arbitrarily short but non-zero simulated time.

In ProModel, "WAIT 0" can be used to put the active entity back on the FEL. It will be returned later (at the same simulated time) by the CUP to the Active State.

In GPSS/H, the active Transaction (Xact) can execute a YIELD Block to migrate immediately from the Active State to the Ready State and force a restart of the CEC scan. Higher-priority (and higher-ranked same priority) Transactions on the CEC then are given a chance to become active before the yielding Transaction itself again becomes active at the same simulated time. (A "PRIORITY PR, YIELD" Block can alternatively be used to reposition the yielding Xact behind other Xacts on the CEC prior to forcing a restart of the CEC scan.)

In SLX there is also a YIELD statement. A normal YIELD shifts the active Puck to the back of its priority class on the CEC and picks up the next Puck. It is also possible to YIELD to a specific other Puck that is on the CEC, in which case the active Puck is not shifted.

In ExtendSim, a message is sent out through the appropriate Block connector when an Item moves into or out of a Block. This message propagates to other connected Blocks, perhaps changing system status or moving Items from one Block to another as a result. When the originating Block eventually receives the reply, it continues processing the original Item. Hence, "yield and then eventually resume" is part of the fabric of ExtendSim's message-based architecture.

\subsection{Conditions Involving the Clock}

Every language provides a time-delay capability for FEL waiting. This works well when an entity needs to wait until a known clock value has been reached. But what if an entity needs to wait for a compound condition involving the clock, such as "wait until my input buffer is empty or it is exactly 5:00 PM?"

A typical approach to this is to clone a dummy ("shadow") entity to do the time-based waiting. Management of such dummy entities can be cumbersome, particularly for very complex rules. ProModel does not use polled waiting, so a dummy entity would be the best approach available. (Otherwise, the condition would not be checked until the other component of the compound condition had a value change.) ExtendSim also does not use polled waiting, so a similar situation applies for Extend. In the ExtendSim architecture this is best described as the use of an additional Block (for example, an Input Data Block) that can schedule an event at the specified time, at which point a message would be sent to the waiting Block. 


\section{Schriber and Brunner}

Even when a polled waiting mechanism is present, if a single entity tries to wait on a compound condition involving the clock, a similar problem can arise. This is because the next polling time may not match the target clock time. SIMAN and AutoMod detect the truth of compound conditions through their end-of-EMP polling mechanisms. GPSS/H also detects the truth through its version of polled waiting (refusal-mode TEST). But in the absence of a clone that waits on the FEL until exactly 5:00 PM (i.e., the same approach as that recommended above with ProModel and Extend), all three of those tools are subject to the possibility that the first EMP that finds the condition true occurs when the clock has a value greater than 5:00 PM. (Also, in the case of AutoMod, the condition is not guaranteed to be checked at the end of the first EMP after 5:00 PM. See the second paragraph of Section 7.1.4.)

SLX recognizes the clock as a related wait-until target. A WAIT UNTIL using a future clock value in a way that contributes to the falseness of the condition will cause the Puck to be scheduled onto the FEL to force an EMP at the precise time referenced. This solves the greater-than-the-desired-time problem. Note that this Puck may also be waiting on one or more delay lists.

\subsection{Mixed-Mode Waiting}

Suppose many entities are waiting to capture a particular resource, while a user-created controller entity is waiting for the condition "shift status is off-shift and number waiting is less than six and resource is not currently in use" to take some action (such as shutting the resource down, in languages that allow user-defined entities to shut down resources; or printing a status message). How can we guarantee that the controller will be able to cut in front of the waiting entities at the appropriate simulated time (before the idle resource is recaptured)?

One way to handle this would be through entity priorities, in languages that offer this mechanism. However, as described below, that may not work even if the controller has higher priority than any other entity.

The key issue is the method used to implement the waiting. If it is "related" for the entities waiting to capture the resource and "polled" for the controller entity waiting for the compound condition (this is what we mean by the term "mixedmode waiting"), things can get complicated. Every time the resource becomes free, a new entity will be selected from a delay list immediately in SIMAN and via the CEL in AutoMod, in both cases preceding the end-of-EMP checking for polled wait conditions (and thereby ignoring the entity priority of the controller). There are ways to work around this if desired, such as using a different type of operation to force a polled wait for entities wishing to use the resource.

In GPSS/H, using a high-priority controller Transaction at a refusal-mode TEST Block, the controller waits at the front of the CEC. The Facility RELEASE will trigger a CEC scan restart and the controller will do its job.

In ProModel there is no polled waiting but there can be related waiting on compound conditions involving Variables. Variables would have to be defined and manipulated for each element of the Boolean condition and, to assure equal competition, the entities waiting to capture the resource might also have to use WAIT UNTIL instead of GET or USE. Another possibility with ProModel would be to have the entity that frees the resource do some state-checking right away (in effect becoming a surrogate for the controller). This is possible because of the deferred-selection method used by ProModel (see Section 8.2).

In the related waiting of SLX, a Puck awaiting a compound condition will be registered on the delay lists of those (and only those) Control Variables that are contributing to the falseness of the condition at the time it is evaluated. The SLX architecture (in which only global or local Control Variables and the clock can be referenced in any sort of conditional wait at the lowest level) assures that there will already be Variables underlying the state changes being monitored. The model developer needs only to be sure they are defined as Control Variables.

As with ProModel and SLX, ExtendSim would use related waiting to detect and immediately respond to a change in the compound condition. The desired effect is achieved in ExtendSim by use of a Program Block, which can be used to issue a message to create a controller Item with its priority set to a value that assures it will be processed before other Items are

processed at a specified simulated time. This Item would wait in ExtendSim's related-waiting fashion (using connectors to monitor the state changes).

\subsection{Interactive Model Verification}

We now comment briefly on why a detailed understanding of "how simulation software works" supports interactive probing of simulation-model behavior.

In general, simulation models can be run interactively or in batch mode. Interactive runs are of use in checking out (verifying) model logic during model building and in troubleshooting a model when execution errors occur. Batch mode is then used to make production runs. 


\section{Schriber and Brunner}

Interactive runs put a magnifying glass on a simulation model while it executes. The modeler can follow the active entity step by step and display the current and future events lists and the delay and user-managed lists as well as other aspects of the model. These activities yield valuable insights into model behavior for the modeler who knows the underlying concepts. Without such knowledge, the modeler might not take full advantage of the interactive tools provided by the software or, worse yet, might even avoid using the tools.

\subsection{Performance Issues}

Simulation experiments can consume substantial amounts of computer time. Other things equal (including the model builder's skill), computer-time requirements depend on the design and implementation of the software used to build models. This dependency can be understood with knowledge of "how simulation software works." For example, consider user-managed lists vs. related waiting in models in which large numbers of entities contend for a resource. Performance is an important enough issue to motivate some simulation software (e.g., ExtendSim; SLX) to supply performance profilers which, for example, can produce histograms showing where CPU time is spent during model execution.

\section{CONFERENCE PRESENTATION}

The presentation of this material at the 2009 Winter Simulation Conference will be based on PowerPoint slides that include use of visuals providing the basis for discussion of the principles presented in this paper. The PowerPoint file can be obtained on request from either of the paper's authors.

\section{ACKNOWLEDGMENTS}

Much of the information in this paper was derived from conversations with software-vendor personnel. The authors gratefully acknowledge the support provided over time by David T. Sturrock, Deborah A. Sadowski, C. Dennis Pegden and Vivek Bapat (SIMAN); Charles Harrell (ProModel); Kenneth Farnsworth and Tyler Phillips (AutoMod); Robert C. Crain and James O. Henriksen (GPSS/H and SLX); and David Krahl (ExtendSim).

\section{REFERENCES}

Diamond, B., J. S. Lamperti, D. Krahl, A. Nastasi, and C. Damiron. 2007. ExtendSim User Guide. San Jose, California: Imagine That Incorporated.

Henriksen, J. O. 2000. SLX: The X is for Extensibility. In Proceedings of the 2000 Winter Simulation Conference, ed. J. A. Joines, R. R. Barton, K. Kang, and P. A. Fishwick, 183-190. Piscataway, New Jersey: Institute of Electrical and Electronics Engineers.

Henriksen, J. O., and R. C. Crain. 2000. GPSS/H: A 23-year retrospective view. In Proceedings of the 2000 Winter Simulation Conference, ed. J. A. Joines, R. R. Barton, K. Kang, and P. A. Fishwick, 177-182. Piscataway, New Jersey: Institute of Electrical and Electronics Engineers.

Kelton, D., R. Sadowski, and N. Swets. 2009. Simulation with Arena, $5^{\text {th }}$ Ed. New York, New York: McGraw Hill.

Krahl, D., and J. S. Lamperti. 1997. A Message-Based Discrete Event Simulation Architecture. In Proceedings of the 1997 Winter Simulation Conference, ed. S. Andradottir, et al, 1361-1367. Piscataway, New Jersey: Institute of Electrical and Electronics Engineers.

Pegden, C. D., R. E. Shannon, and R. P. Sadowski. 1995. Introduction to Simulation Using SIMAN. New York, New York: McGraw-Hill.

Phillips, T. 1997. Know your AutoMod Current Events. In AutoFlash, 10(7). Bountiful, Utah: AutoSimulations, Inc.

ProModel Corporation. 1995. ProModel Version 3 User Guide. Orem, UT: ProModel Corporation.

ProModel Corporation. 2008. ProModel Version 7.5 User Guide. Orem, UT: ProModel Corporation.

Schriber, T. J., and D. T. Brunner. 1996. Inside Simulation Software: How It Works and Why It Matters. (This paper was chosen at the 40th Anniversary WSC in 2007 as one of ten "landmark papers" given during the first forty years of the WSC.) In Proceedings of the 1996 Winter Simulation Conference, ed. J. Charnes, D. Morrice, D. Brunner, and J. Swain, 23-30. Piscataway, New Jersey: Institute of Electrical and Electronics Engineers.

Schriber, T. J. and D. T. Brunner. 1998. How Discrete-Event Simulation Software Works. Chapter 24 in Handbook of Simulation: Principles, Methodology, Advances, Applications, and Practice, ed. J. Banks. New York, New York: John Wiley $\&$ Sons.

Schulze, T. 2008. Simulation Needs SLX. Annandale, Virginia: Wolverine Software. 
Swain, J. J. 2007. New Frontiers in Simulation (Biennial survey of discrete-event simulation software). OR/MS Today 34(5): 32-43. Baltimore, Maryland: INFORMS. (The next biennial survey will be available in OR/MS Today in late 2009.)

\section{AUTHOR BIOGRAPHIES}

THOMAS J. SCHRIBER is a Professor of Business Information Technology at The University of Michigan. He is a recipient of the INFORMS Simulation Society's Lifetime Professional Achievement Award and of the Society's Distinguished Service Award, and has received the James R. Wilson Winter Simulation Conference Board of Directors' Award for Distinguished Service. He is a Charter Member and Fellow of the Decision Sciences Institute and author of Simulation Using GPSS, which saw widespread use for many years. He has been a WSC Program Chair and served ten years on the WSC Board of Directors, chairing the board for two years. He is a member of ASIM (the German-language simulation society), the Decision Sciences Institute, INFORMS, and the Institute of Industrial Engineers. His email and web addresses are: <schriber@umich.edu> and <http: / /www.bus.umich.edu/>.

DANIEL T. BRUNNER is Vice President of System Analysis at Kiva Systems, Inc., a provider of innovative material handling systems for order fulfillment and warehousing. He holds a B.S.E.E. from Purdue University and an MBA from The University of Michigan. He has served as the Winter Simulation Conference Business Chair and General Chair and as the Transportation Applications Track Coordinator. His email and web addresses are: <dbrunner@kivasystems . com>and $<$ http://www.kivasystems.com>. 